\title{
Evaluation of local carotid stiffness and inflammatory biomarkers in stable angina pectoris
}

\author{
Abdullah Nabi Aslan ${ }^{1}$, Ayşe Nur Özcan², Hüseyin Ayhan³ ${ }^{3}$ Serkan Sivri ${ }^{1}$, Filiz Çelebi ${ }^{3}$, Karabekir Ercan², \\ Tahir Durmaz ${ }^{3}$ \\ ${ }^{1}$ Department of Cardiology, Atatürk Education and Research Hospital, Ankara, Turkey \\ 2Department of Radiology, Atatürk Education and Research Hospital, Ankara, Turkey \\ ${ }^{3}$ Department of Cardiology, Faculty of Medicine, Yildirim Beyazit University, Ankara, Turkey
}

Adv Interv Cardiol 2017; 13, 2 (48): 122-129

DOI: https://doi.org/10.5114/pwki.2017.68046

\begin{abstract}
Introduction: Arterial stiffness (AS) is a well-accepted and reliable predictor of atherosclerotic diseases. Inflammation plays an important role in the development of AS.

Aim: To evaluate local carotid stiffness (CS) together with fibrinogen and high-sensitivity C-reactive protein (hsCRP) levels in stable angina pectoris (SAP) patients.

Material and methods: The study consisted of 353 consecutive patients with SAP. All underwent coronary angiography (CAG) after the evaluation of local CS parameters and carotid intima-media thickness (IMT) from both common carotid arteries by a realtime echo-tracking system. Baseline inflammatory biomarkers, serum hsCRP and fibrinogen levels were measured. Based on CAG findings, the patients were classified into 4 groups: control subjects with normal coronary arteries (group $1, n=86$ ), single-vessel disease (group 2, $n=104$ ), double-vessel disease (group 3, $n=95$ ) and triple-vessel disease (group 4, $n=68$ ).

Results: The mean carotid pulse wave velocity (PWV) in patients with angiographically confirmed coronary artery disease (CAD) was significantly higher than that in patients with normal coronary arteries $(7.82 \pm 1.76 \mathrm{vs} .6 .51 \pm 0.85 \mathrm{~cm} / \mathrm{s}, p=0.001)$. The mean carotid IMT was detected to be significantly higher in group 4 patients compared to those in group $1(p<0.001)$ and group 2 $(p=0.001)$. Significant correlations were observed between both inflammatory biomarkers and the number of diseased vessels and carotid PWV. Using multi-variate analysis, carotid stiffness, carotid IMT, hsCRP and fibrinogen were independently associated with the presence and extent of CAD.

Conclusions: Local CS, carotid IMT, hSCRP and fibrinogen levels are significant predictors of atherosclerotic burden and they may facilitate the identification of high-risk patients for the early diagnosis and prompt treatment of CAD.
\end{abstract}

Key words: stable angina pectoris, local carotid stiffness, pulse wave velocity, intima-media thickness, high-sensitivity C-reactive protein, fibrinogen.

\section{Introduction}

There is a variety of evidence on the crucial role of pre-clinical markers in determining the risk of cardiovascular (CV) morbidity and mortality in individuals. Arterial stiffness (AS) parameters are among these markers that are commonly used to identify structural and functional changes in the arteries during the atherosclerotic process $[1,2]$. Diverse clinical studies have demonstrated the independent predictive value of AS for CV events in patients with hypertension (HT), diabetes mellitus (DM), end-stage renal disease, in elderly patients and in the general population [1, 3-6]. Based on this evidence, AS is considered a useful marker in the assessment of CV risk and diagnosis of subclinical CV disease [7]. Arterial stiffness can be measured locally in the common carotid artery (CCA) through carotid pulse wave velocity (PWV). Measurement of PWV is the simplest non-invasive, rapid and widely used method in the evaluation of large AS [8]. The localized PWV of an artery has a direct association with elastic modulus [9]. Recently, several methods have emerged for the estimation of PWV using ultrasound principles [1]. Echo-tracking systems (ETS) - high-resolution wall-tracking systems that analyze the raw radiofrequency (RF) signals - have been developed [10]. They provide optimal conditions for the precise determination

\section{Corresponding author:}

Abdullah Nabi Aslan, Department of Cardiology, Atatürk Education and Research Hospital, Bilkent 3, Cankaya, 06800 Ankara, Turkey, phone: +90 5327801305, e-mail: drnabiaslan@hotmail.com

Received: 23.10.2016, accepted: 7.02.2017. 
of local AS, which is directly determined, requiring no assumption from models of circulation [1]. These systems are able to track the arterial wall movement in real time and to measure the carotid arterial distension with an error less than $5 \mu \mathrm{m}$ [11]. They also have the advantage of automatic measurement of carotid intima-media thickness (IMT) simultaneously with carotid PWV. Carotid IMT reflects the presence of atherosclerotic plaques and the general atherosclerotic burden [12, 13]. Carotid IMT evaluation by ultrasound allows an easy, safe, non-invasive and cost-effective method to detect subclinical atherosclerosis and has been shown to be a prognosticator of CV events [14].

With the recognition of the crucial link between arterial damage, inflammation and coronary atherosclerosis, inflammatory biomarkers such as high-sensitivity C-reactive protein (hsCRP) and fibrinogen estimation have assumed a vital role in cardiac risk assessment. There is an increasing amount of evidence that hsCRP is increased in patients with CV disease, and elevated levels of this marker usually precede the development of CV disease [15]. Among patients with stable angina pectoris (SAP), hsCRP levels have been related to an increased vascular event rate [16]. Fibrinogen is also an important inflammatory biomarker in addition to its crucial role in coagulation. Previous studies have found that the incidence of coronary artery atherosclerosis and CAD increases significantly as the level of fibrinogen becomes elevated $[17,18]$.

\section{Aim}

The present study aimed to study local CS that was measured by a high-spatial resolution ETS (MyLab25; ESAOTE, Genoa, Italy) in addition to hsCRP and fibrinogen levels among patients presenting with SAP. This is the first study evaluating the association between local CS parameters and fibrinogen level in SAP patients.

\section{Material and methods}

\section{Study population}

The study was designed as an observational (casecontrol) study and included 353 consecutive patients (age 29 to 88 years) hospitalized due to the complaint of SAP in our clinic between November 2013 and March 2016. The diagnosis of SAP was confirmed based on a proper history, a detailed physical examination, 12lead resting electrocardiography and if necessary stress tests such as the exercise treadmill test, stress echocardiography or myocardial perfusion scintigraphy. Patients with a previous history of CV disease (e.g. coronary artery disease, carotid artery disease, peripheral artery disease, stroke, transient ischemic attack), cardiac arrhythmia, valvular heart disease, heart failure, chronic inflammatory disorders, renal dysfunction (estimated glomerular filtration rate $(e G F R)<60 \mathrm{ml} / \mathrm{min} / 1.73 \mathrm{~m}^{2}$ ) and using statins were excluded from the study. All patients also had a transthoracic echocardiography examination and routine biochemical analysis of the blood. Blood samples were taken from the antecubital vein after 8-hour overnight fasting. All patients underwent laboratory examinations including total cholesterol (TC), triglycerides (TG), high-density lipoprotein (HDL) cholesterol, low-density lipoprotein (LDL) cholesterol, fasting blood glucose, blood urea nitrogen (BUN), serum creatinine, fibrinogen and hsCRP levels. The serum hsCRP levels were measured with the immunoturbidimetric method (Roche Diagnostics $\mathrm{GmbH}$ ). The mean concentration range was accepted as $0-1.0 \mathrm{mg} / \mathrm{l}$. Fibrinogen levels were measured with the coagulation test in the plasma (STA, Diagnostica Stago, Asnieres sur Seine Cedex, France) and the mean concentration range was accepted as $185-450 \mathrm{mg} / \mathrm{dl}$. All patients were studied by the Esaote wall tacking system (MyLab25; ESAOTE, Genoa, Italy) at the Department of Radiology. All measurements were obtained from both common carotid arteries by a single experienced operator and recorded. After that, elective coronary angiography (CAG) was performed using the Judkins technique on digitized coronary angiography equipment (Siemens, Artis Zee, Germany) among all patients. Patients were divided into 4 groups based on their CAG findings. Group 1 $(n=86)$ included patients with normal coronary arteries with no lesion in any coronary arteries, group $2(n=104)$ included patients with a single-vessel disease that was defined as $\geq 70 \%$ luminal obstruction in a vessel with a diameter of $>2 \mathrm{~mm}$ or more, group $3(n=95)$ included patients with double-vessel disease, and group $4(n=68)$ included patients with triple-vessel disease. The study protocol was confirmed by the local ethics committee of our hospital and written informed consent of all patients was taken.

\section{Assessment of local carotid arterial stiffness}

For the evaluation, subjects lay down in the supine position in a quiet and temperature-controlled room $\left(23^{\circ} \mathrm{C}\right)$ for $10 \mathrm{~min}$. An independent radiologist trained and certified in vascular echography performed blood pressure (BP) and arterial measurements. Before the ultrasound examination, BP measurements (both systolic blood pressure (SBP) and diastolic blood pressure (DBP)) of the patients were taken from the dominant arm by an aneroid sphygmomanometer at 5-minute intervals for $15 \mathrm{~min}$ and the average was taken as the casual BP level. Mean blood pressure (MBP) was calculated as: $M B P=D B P+[(S B P-D B P) / 3]$. Brachial pulse pressure (PP) was calculated as: $\mathrm{PP}=\mathrm{SBP}-\mathrm{DBP}$.

All patients underwent common carotid artery (CCA) measurements with a novel system (MyLab25 QAS machine; ESAOTE, Genoa, Italy) based on an automatic high resolution echo-tracking technology (WallTrack system) that utilized a 7.5-12 MHz linear array transducer. The systolic and diastolic carotid diameters were automati- 


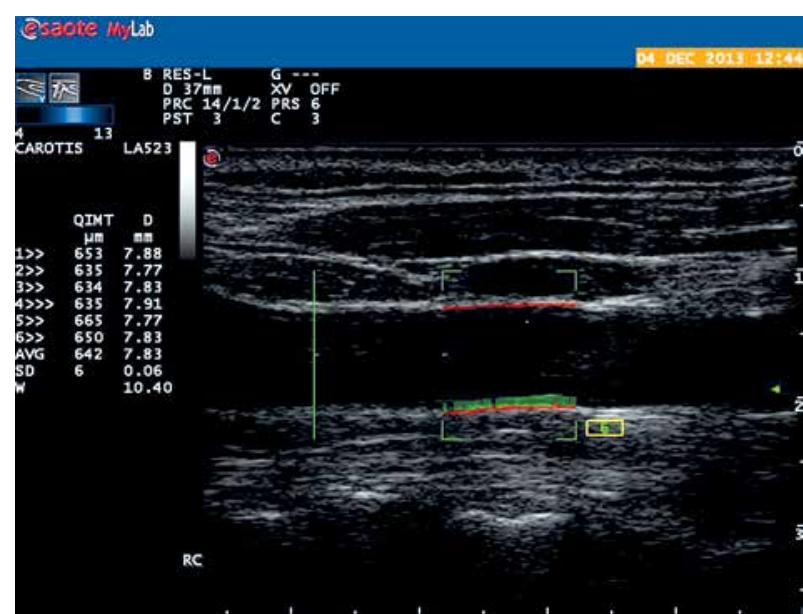

Figure 1. Measurement of carotid intima-media thickness by Esaote wall tracking (QAS, MyLab) system. The movement of carotid walls is tracked in the entire region of interest (green rectangle) composed of 32 scanning lines. Continuous red lines indicate the automatic positioning of wall-tracking points at the media-adventitia interface. Continuous green lines show dynamically the amplified vessel wall movement. The values of carotid intima-media thickness (QIMT) and minimum diameter (D) are displayed on the screen, and the average value (AVG) over the last six beats and SD are continuously calculated

cally measured on the distal wall of the left and right CCA $2 \mathrm{~cm}$ below the bifurcation. Concisely, variations in momentary arterial diameter during the cardiac cycles were displayed in real time on a graphic giving the operator visual feedback to adjust the quality of the image before measurement acquisition (Figure 1). Carotid diameter was calculated as the distance between media-adventitia interfaces. Systolic and diastolic values were determined for each cardiac cycle and carotid distension was calculated as the mean value of their difference in the last $8 \mathrm{~s}$ of the image sequences. The cross-sectional distensibility coefficient (DC) was estimated through the variations in arterial cross-sectional area and blood pressure during systole as described previously [19]. Distensibility coefficient was calculated as $D C=\Delta A /(A \times \Delta P)$, where $A$ is the diastolic lumen area, $\Delta \mathrm{A}$ is the stroke change in lumen area, and $\Delta \mathrm{P}$ is local PP. Lumen area and stroke change in lumen area were calculated from diameter and distension values, assuming the cross section of the artery to be circular. Finally, DC was converted into carotid stiffness (CS) using the Moens-Korteweg equation, $P W V=[(P P \times A) /(\Delta A \times \rho)]^{1 / 2}$, where $\rho$ is the blood density. This formula gives $C S=(\rho \times D C)^{-1 / 2}$, expressed in $\mathrm{m} / \mathrm{s}$, allowing a direct comparison with carotid-femoral PWV. Common carotid IMT was automatically and simultaneously measured as the mean of the relative values of the last $8 \mathrm{~s}$ on the same image sequences. Carotid IMT was measured in the far wall of the CCA, $1 \mathrm{~cm}$ proximal to the carotid bulb. Parameters are indicated as the mean of the right and left CCA value.

\section{Statistical analysis}

Statistical analysis was performed using SPSS for Windows, version 20 (IBM corporation, Armonk, NY). Continuous variables were expressed as mean \pm SD and categorical variables as percentage. Comparison of parametric values between the four groups was carried out by means of a two-tailed Student's $t$-test. The Bonferroni correction was used to compare the variables in four groups. Post-hoc analysis was done for multiple comparison between all groups as well. Multivariate logistic regression analysis was performed to evaluate the impact of clinical and biochemical factors on coronary artery disease. A $p$-value $<0.05$ was considered statistically significant.

\section{Results}

\section{Patients characteristics}

Subjects enrolled in the present study were divided into four groups based on their CAG findings. The demographic features and baseline clinical characteristics of these groups are summarized in Table I. The study population overall included 274 males and 79 females with a mean age of $59.2 \pm 10.5$ (range: $29-88$ ) years. There were no significant differences between the groups with respect to age, weight, height, body mass index (BMI), SBP, brachial PP, MBP, heart rate, prevalence of $H T$, DM, hyperlipidemia, TC, LDL cholesterol, TG, BUN and creatinine levels and eGFR ( $p>0.05$ for all). A statistically significant male predominance was present in group 2 $(p=0.005)$ and group $4(p=0.007)$ patients compared to those in group 1. Although the SBP was similar between all groups, the DBP was shown to be significantly lower in patients with CAD compared to the patients with normal coronary arteries (79.6 \pm 17.2 vs. $88.2 \pm 16.9 \mathrm{~mm} \mathrm{Hg}$, respectively, $p=0.012$ ). We found a higher proportion of current smokers in CAD groups compared to group 1 (59.7\% vs. $37.2 \%$, respectively, $p=0.014$ ). HDL-cholesterol level was significantly higher in group 1 patients in comparison with group 2 ( $49.3 \pm 16.5$ vs. $41.0 \pm 10.4 \mathrm{mg} / \mathrm{dl}$, respectively, $p=0.046)$ and group 3 (49.3 \pm 16.5 vs. 40.3 $\pm 10.0 \mathrm{mg} / \mathrm{dl}$, respectively, $p=0.046$ ) patients. The mean hsCRP level was detected to be significantly higher in patients with CAD compared to patients with normal coronary arteries (1.43 \pm 1.8 vs. $0.44 \pm 0.4 \mathrm{mg} / \mathrm{l}$, respectively, $p=0.004)$. When we evaluated the hsCRP levels between the groups, we found that hsCRP level was significantly higher in group $2(p=0.035)$, group 3 ( $p=$ $0.006)$ and group $4(p=0.010)$ patients than those in group 1 . The mean fibrinogen level was also significantly higher in CAD groups compared to controls with normal coronary arteries (294.3 \pm 57.3 vs. $264.8 \pm 44.3 \mathrm{mg} / \mathrm{dl}$, 
Table I. Clinical characteristics of the study population

\begin{tabular}{|c|c|c|c|c|c|}
\hline Variables & Group $1(n=86)$ & Group $2(n=104)$ & Group $3(n=95)$ & Group $4(n=68)$ & $P$-value \\
\hline Age [years] & $61.4 \pm 10.8$ & $57.2 \pm 12.1$ & $59.7 \pm 10.4$ & $61.4 \pm 9.1$ & 0.149 \\
\hline Gender, male (\%) & $47(54.6)$ & $91(87.5)$ & 75 (78.9) & $60(88.2)$ & 0.003 \\
\hline Weight [kg] & $82.6 \pm 17.2$ & $85.8 \pm 14.6$ & $82.4 \pm 11.1$ & $77.9 \pm 10.4$ & 0.156 \\
\hline Height [m] & $1.67 \pm 0.10$ & $1.69 \pm 0.10$ & $1.69 \pm 0.08$ & $1.65 \pm 0.14$ & 0.317 \\
\hline BMI $\left[\mathrm{kg} / \mathrm{m}^{2}\right]$ & $29.8 \pm 8.5$ & $29.7 \pm 4.5$ & $29.1 \pm 3.2$ & $28.3 \pm 3.4$ & 0.322 \\
\hline $\mathrm{SBP}[\mathrm{mm} \mathrm{Hg}]$ & $132.8 \pm 25.7$ & $132.4 \pm 16.4$ & $130.3 \pm 21.3$ & $127.9 \pm 12.6$ & 0.276 \\
\hline $\mathrm{DBP}[\mathrm{mm} \mathrm{Hg}]$ & $88.2 \pm 16.9$ & $78.5 \pm 15.1$ & $82.7 \pm 17.6$ & $77.1 \pm 12.9$ & 0.036 \\
\hline Brachial PP [mm Hg] & $45.6 \pm 17.4$ & $45.9 \pm 15.2$ & $48.6 \pm 14.4$ & $49.4 \pm 13.7$ & 0.542 \\
\hline $\mathrm{MBP}[\mathrm{mm} \mathrm{Hg}]$ & $103.2 \pm 18.6$ & $93.4 \pm 13.2$ & $98.6 \pm 17.5$ & $92.7 \pm 11.6$ & 0.067 \\
\hline Heart rate [bpm] & $75.7 \pm 12.3$ & $77.8 \pm 12.0$ & $72.8 \pm 10.4$ & $75.7 \pm 13.4$ & 0.172 \\
\hline Current smokers, $n(\%)$ & $32(37.2)$ & $73(70.1)$ & $52(54.7)$ & $44(64.7)$ & 0.014 \\
\hline HT, $n(\%)$ & $58(67.4)$ & $76(73.4)$ & 75 (78.9) & $59(86.7)$ & 0.248 \\
\hline $\mathrm{DM}, n(\%)$ & $23(26.7)$ & $42(40.3)$ & $27(28.4)$ & $30(44.1)$ & 0.541 \\
\hline HL, $n(\%)$ & $32(37.2)$ & $62(59.6)$ & $57(60.0)$ & $38(55.8)$ & 0.133 \\
\hline $\mathrm{TC}[\mathrm{mg} / \mathrm{dl}]$ & $194.3 \pm 29.1$ & $187.3 \pm 53.9$ & $183.2 \pm 39.0$ & $172.9 \pm 53.4$ & 0.408 \\
\hline LDL-C [mg/dl] & $111.1 \pm 28.0$ & $110.8 \pm 45.3$ & $108.0 \pm 38.4$ & $97.9 \pm 41.1$ & 0.616 \\
\hline $\mathrm{HDL}-\mathrm{C}[\mathrm{mg} / \mathrm{dl}]$ & $49.3 \pm 16.5$ & $41.0 \pm 10.4$ & $40.3 \pm 10.0$ & $44.3 \pm 13.1$ & 0.029 \\
\hline $\mathrm{TG}[\mathrm{mg} / \mathrm{dl}]$ & $174.0 \pm 90.0$ & $182.1 \pm 115.1$ & $172.6 \pm 78.8$ & $148.7 \pm 85.4$ & 0.634 \\
\hline $\mathrm{BUN}[\mathrm{mg} / \mathrm{dll}]$ & $42.8 \pm 18.0$ & $36.9 \pm 13.6$ & $38.9 \pm 17.5$ & $43.1 \pm 13.2$ & 0.366 \\
\hline Creatinine $[\mathrm{mg} / \mathrm{dl}]$ & $0.9 \pm 0.3$ & $0.8 \pm 0.2$ & $1.0 \pm 0.5$ & $1.0 \pm 0.1$ & 0.234 \\
\hline $\mathrm{eGFR}\left[\mathrm{mL} / \mathrm{min} / 1.73 \mathrm{~m}^{2}\right]$ & $79.2 \pm 28.0$ & $84.3 \pm 27.9$ & $94.7 \pm 60.2$ & $79.3 \pm 24.9$ & 0.396 \\
\hline hsCRP level [mg/l] & $0.44 \pm 0.4$ & $0.91 \pm 1.0$ & $1.75 \pm 2.2$ & $1.79 \pm 1.2$ & 0.001 \\
\hline Fibrinogen [mg/dl] & $264.8 \pm 44.3$ & $272.4 \pm 48.6$ & $294.0 \pm 58.5$ & $317.3 \pm 66.4$ & 0.012 \\
\hline
\end{tabular}

Data are expressed as mean $\pm S D$. BMI - body mass index, BUN - blood urea nitrogen, DBP - diastolic blood pressure, DM - diabetes mellitus, eGFR - estimat ed glomerular filtration rate, HDL-C - high-density lipoprotein cholesterol, HL - hyperlipidemia, hSCRP - high-sensitivity C-reactive protein, HT - hypertension, $L D L-C$ - low-density lipoprotein cholesterol, MBP - mean blood pressure, PP-pulse pressure, SBP-systolic blood pressure, TC - total cholesterol, TG - triglyceride.

respectively, $p=0.014$ ). Plasma fibrinogen levels demonstrated a stepwise increase from the control group to the triple-vessel group. However, a statistically significant increase was only observed in group $3(p=0.024)$ and group $4(p<0.001)$ but not in group $2(p=0.121)$ patients compared to controls with normal coronary arteries.

Local CS parameters measured by RF-based ETS are demonstrated in Table II. Accordingly, the mean values of carotid PP, carotid diameter and carotid distension

Table II. Pulse wave velocity and other echo-tracking parameters in the study population

\begin{tabular}{|c|c|c|c|c|c|}
\hline Arterial parameters & Group $1(n=86)$ & Group $2(n=104)$ & Group $3(n=95)$ & Group $4(n=68)$ & $P$-value \\
\hline Carotid pulse pressure [mm Hg] & $39.7 \pm 15.3$ & $37.4 \pm 12.7$ & $40.6 \pm 12.1$ & $43.4 \pm 12.2$ & 0.385 \\
\hline Carotid IMT [mm] & $0.67 \pm 0.13$ & $0.75 \pm 0.15$ & $0.80 \pm 0.17$ & $0.89 \pm 0.29$ & $<0.001$ \\
\hline Carotid diameter [mm] & $8.33 \pm 0.7$ & $8.44 \pm 0.9$ & $8.46 \pm 0.8$ & $8.43 \pm 1.1$ & 0.772 \\
\hline Carotid distension [mm] & $0.33 \pm 0.11$ & $0.38 \pm 0.17$ & $0.36 \pm 0.12$ & $0.39 \pm 0.16$ & 0.439 \\
\hline Carotid PWV [m/s] & $6.51 \pm 0.85$ & $7.27 \pm 1.8$ & $7.68 \pm 1.6$ & $8.12 \pm 2.1$ & 0.007 \\
\hline
\end{tabular}

Data are expressed as mean $\pm S D$. IMT - intima-media thickness, PWV-pulse wave velocity. 
were similar between groups $(p=0.385, p=0.772, p=$ 0.439 , respectively). However, the mean value of carotid PWV was significantly higher in CAD patients compared to patients with normal coronary arteries (7.82 \pm 1.76 vs. $6.51 \pm 0.85 \mathrm{~m} / \mathrm{s}$, respectively, $p=0.001$ ). When it was compared between all the groups, it was found that carotid PWV was significantly higher in group 2 $(p=0.031)$, group $3(p=0.033)$ and group $4(p=0.007)$ patients than in subjects with normal coronary arteries (group 1).

The correlations between AS and various demographic and clinical characteristics are presented in Table III. According to these results, there was a significant positive correlation between AS and age $(r=0.413$, $p<0.001)$, DM $(r=0.612, p<0.001)$, SBP $(r=0.422$, $p<0.001)$, brachial PP $(r=0.343, p<0.001)$, carotid PP $(r=0.461, p<0.001)$ and mean BP $(r=0.311, p=0.001)$, whereas there was a significant negative correlation between AS and eGFR $(r=-0.245, p=0.005)$. Bivariate analysis showed a significant correlation between carotid PWV and hsCRP $(r=0.214, p=0.025)$ and fibrinogen $(r=0.411, p=0.003)$ levels. There was also a strong positive correlation between carotid IMT and hsCRP level $(r=0.476, p=0.008)$. Moreover, we confirmed a positive

Table III. Correlation between clinical characteristics and arterial stiffness in study population $(N=353)$

\begin{tabular}{lcc} 
Risk factors & Arterial stiffness (pulse wave velocity) \\
\cline { 2 - 3 } & $\boldsymbol{r}$ & $\boldsymbol{p}$ \\
\hline Age & 0.413 & $<0.001$ \\
\hline Body mass index & 0.210 & 0.242 \\
\hline Diabetes mellitus & 0.612 & $<0.001$ \\
\hline Systolic BP & 0.426 & $<0.001$ \\
\hline Diastolic BP & 0.185 & 0.054 \\
\hline Brachial PP & 0.343 & $<0.001$ \\
\hline Carotid PP & 0.461 & $<0.001$ \\
\hline Mean BP & 0.311 & 0.001 \\
\hline Total cholesterol & 0.061 & 0.605 \\
\hline HDL cholesterol & 0.147 & 0.107 \\
\hline LDL cholesterol & 0.045 & 0.634 \\
\hline Triglyceride & 0.107 & 0.221 \\
\hline eGFR & -0.245 & 0.005 \\
\hline C-IMT & 0.312 & 0.008 \\
\hline hsCRP & 0.214 & 0.025 \\
\hline Fibrinogen & 0.411 & 0.003 \\
\hline BP- blood press & & 01 \\
\hline
\end{tabular}

$B P$-blood pressure, C-IMT-carotid intima-media thickness, $H D L$ - high-density lipoprotein, hSCRP - high-sensitivity C-reactive protein, $L D L$ - low-density lipoprotein, eGFR - estimated glomerular filtration rate. correlation between CS and intima-media thickness ( $r=$ $0.312, p=0.008$ ) in the whole study population.

All the factors of significant difference in the univariate analysis and the traditional risk factors of CAD were entered in the multivariate logistic regression analysis to detect factors that were significantly associated with CAD. As a result, age, male gender, DM, hsCRP, fibrinogen, carotid PWV and carotid IMT showed a significant association with the occurrence of CAD (Table IV).

\section{Discussion}

This study evaluated AS parameters using carotid PWV by the local measurement method on the carotid arterial wall in SAP patients. Additionally, hsCRP and fibrinogen levels were measured to investigate the effect of inflammation on CS parameters and the severity and extent of CAD. As a result, a positive association between carotid PWV, intima-media thickness, hsCRP and fibrinogen levels and the extent of CAD was found. In addition, a significant positive correlation was detected between CS, carotid IMT, fibrinogen and hsCRP levels.

The pathophysiology of the coronary atherosclerotic process is complex, involving endothelial cell injury, lipid infiltration and conversion of macrophages into lipid-lad-

Table IV. Multivariate logistic regression analysis of factors related to coronary artery disease

\begin{tabular}{|c|c|c|c|}
\hline Parameter & Odds ratio & $\begin{array}{c}95 \% \\
\text { confidence } \\
\text { interval }\end{array}$ & $P$-value \\
\hline Age & 1.013 & $0.668-0.819$ & $0.039^{*}$ \\
\hline Male & 1.596 & $1.166-1.489$ & $0.042^{\star}$ \\
\hline BMI & 0.996 & $0.929-1.106$ & 0.349 \\
\hline Hypertension & 0.645 & $0.384-1.163$ & 0.128 \\
\hline Diabetes & 2.127 & $1.244-3.698$ & $0.004^{*}$ \\
\hline Smoking & 1.174 & $0.820-2.129$ & 0.385 \\
\hline Dyslipidemia & 1.752 & $1.153-2.870$ & 0.051 \\
\hline Total cholesterol & 1.014 & $0.998-1.028$ & 0.688 \\
\hline LDL cholesterol & 1.005 & $0.996-1.013$ & 0.547 \\
\hline HDL cholesterol & 0.986 & $0.974-1.024$ & 0.641 \\
\hline Triglyceride & 1.024 & $0.995-1.102$ & 0.302 \\
\hline hsCRP & 0.918 & $0.853-0.987$ & $0.028^{*}$ \\
\hline Fibrinogen & 1.014 & $1.124-1.362$ & $0.001^{*}$ \\
\hline Carotid PWV & 1.084 & $1.011-1.164$ & $0.034^{*}$ \\
\hline C-IMT & 1.215 & $1.052-1.985$ & $0.016^{*}$ \\
\hline
\end{tabular}

"Significant difference. BMI - body mass index, C-IMT - carotid intima-media thickness, HDL - high-density lipoprotein, hSCRP - high-sensitivity C-reactive protein, $L D L$-low-density lipoprotein, PWV-pulse wave velocity. 
en foam cells which closely correlate with inflammation [20]. The inflammatory response is a crucial point in the occurrence, development and progression of CAD. Therefore, serum levels of inflammatory markers can give a clue about the disease severity and may be used to predict acute coronary events [21]. In the present study, we found that the serum hsCRP and fibrinogen levels were related to CAD severity as higher serum levels of fibrinogen and hsCRP were determined in patients with single-, double- and triple-vessel disease. Liang et al. [22] also observed significantly higher hsCRP levels in patients with double- and triple-vessel disease. Current studies suggest that fibrinogen similar to hsCRP is an important inflammatory factor [23]. Therefore, an increase in fibrinogen level enhances the risks of atherosclerosis and CV events $[17,18]$. The Atherosclerosis Risk in Communities Study showed that elevated levels of fibrinogen are a risk factor in CAD. Karahan et al. [24] also showed serum fibrinogen level as a significant inflammatory marker for predicting the severity of CAD. Similar to these studies, plasma fibrinogen levels also demonstrated a stepwise increase from the control group to the triple-vessel group in our study. Moreover, a positive correlation between fibrinogen and hSCRP levels and carotid PWV was detected. These data indicate that AS is correlated with systemic inflammation and the inflammation plays an important role in the development of arterial wall stiffness. Increased AS is considered as one of the key factors related to CV disease $[25,26]$. It causes an increase in cardiac ventricular load, thereby reducing cardiac systolic performance and increasing myocardial oxygen demand [27]. This may lead to the progression of atherosclerosis, likely mediated by increased cycle stress resulting in arterial wall thickening. Previous studies have shown that increased AS prevents the hemodynamic buffering effect of the CV system, contributing to elevated SBP and PP, CAD and left ventricular hypertrophy $[25,28]$. The PWV is defined as the velocity at which the pressure waves, generated by the systolic contraction of the heart, propagate along the arterial tree. The evaluation of PWV provides complementary information about the elastic properties of the arterial system. The higher PWV corresponds to lower vessel distensibility and compliance and, therefore, to higher AS. Increased PWV is associated with a significant CV risk in the general population $[29,30]$. It is an important marker of AS and is now widely accepted as the gold standard measure of AS [1]. There are various invasive and non-invasive methods in determining PWV. The non-invasive measurement of AS is usually accomplished by a set of devices that measure PWV and that provide the capability to perform pulse pressure waveform analysis. Because, the local PWV measurement is made on a short segment of an artery and represents an early diagnostic tool able to identify the local stiffness of the arterial wall [31]. A non-invasive color Doppler ultrasound machine was used in this study to measure local
CS. The CS is usually determined cross-sectionally from local changes in pressure and artery diameter [32]. On clinical application, in the early stage of atherosclerotic disease, the elastic properties will be affected locally and thus the functional properties of the local large arteries can be analyzed with local assessment of PWV [33]. Gaszner et al. [34] reported that carotid PWV in CAD patients was significantly higher than the control group with normal coronary arteries ( $7.4 \pm 1.3$ vs. $6.5 \pm 1.1 \mathrm{~m} / \mathrm{s}, p<0.01)$. Similar findings were detected in the present study.

Another finding of this study concerns the observation that carotid IMT, measured simultaneously on the same image sequence and on the same tract of CCA, was significantly greater in patients with triple-vessel disease as compared to other groups. There are many reports determining the association between carotid IMT and atherosclerotic lesions in a single arterial territory such as coronary, renal or lower limb arteries [35-37]. In another study, the mean carotid IMT was detected to be increased with greater atherosclerosis extent, and it may be translated into the number of territories with significant stenosis [12]. Additionally, there was a significant positive correlation between carotid IMT and hsCRP level and carotid PWV. Kablak-Ziembicka et al. [38] investigated the relationship between carotid IMT, inflammatory biomarkers and atherosclerotic extent. Similarly to our results, they found a significant relation between carotid IMT value and atherosclerosis extent. Moreover, they observed more advanced atherosclerosis with a higher baseline hsCRP level. The results of the Three-City Study documented the importance of the simultaneous measurement of both CS and carotid IMT in the same vessel [39]. According to this study, an association between CS and carotid IMT was detected, similarly to our study. Carotid IMT and CS provide structural and functional data, respectively, and assessment of both parameters gives complementary information on the carotid vessel.

There was not a significant relationship of PWV with the lipid profile of the patients including TC, LDL cholesterol, HDL cholesterol and TG in this study. Although positive and negative relations of AS with cholesterol level have been reported using different methods, most studies using PWV have determined no correlation with TC $[28,40]$. Our findings were in agreement with a recent large population-based study by Amar et al. [28]. They detected no significant association between PWV and TC, TG and HDL cholesterol as well.

On the other hand, a significantly negative correlation was determined between PWV and eGFR. AS is a highly prevalent condition in patients with chronic kidney disease, and recent studies showed the independent impact of AS on mortality and morbidity in this patient population [41]. Sedaghat et al. studied 3666 patients and also obtained results similar to our study that higher indices of AS which was evaluated using PWV are related to a steeper decline in kidney function [42]. 
All these results are in line with the previous data. However, to the best of our knowledge, this is the first study evaluating the association between fibrinogen level and local CS parameters in SAP patients. Also, AS parameters were obtained from a novel RF-based technique requiring specific and sophisticated ultrasound equipment. A standard high definition ultrasound machine that automatically measures carotid PWV and IMT, both of which are pre-clinical indicators of atherosclerosis, was used for early prediction of CAD. The RF-based ETS implemented in standard ultrasound equipment may provide a quick and simple tool to evaluate local AS during routine measurements of IMT. Such a combined evaluation of local arterial structure and function may further improve the assessment of vascular aging and early atherosclerotic injury.

There are some limitations of this study. First, our study groups were composed of a relatively small number of individuals. Second, we used brachial BP measurement for the calibration of carotid diameter changes. Brachial pressure usually overestimates the central pressure, especially in young subjects [43]. Third, carotid artery elastic parameters were measured locally at a specific site; therefore stiffening of the carotid arterial wall can be attributed to an anatomically defined segment of the arterial tree. Hence, further large-scale studies are required to generalize the clinical significance of our findings and to use ETS in routine clinical practice for the assessment of vascular function and CAD risk.

\section{Conclusions}

Carotid stiffness and carotid IMT measured using the echo-tracking technique in addition to fibrinogen and hsCRP levels were well correlated with the presence and extent of CAD in patients with SAP. Thus, local carotid arterial stiffness and function measurements together with fibrinogen and hsCRP level may provide additional benefit in clinical practice for the evaluation of patients suspected to have CAD. Therefore, this novel method together with measurement of the above inflammatory biomarkers may be used to detect high-risk SAP patients and accordingly to plan the clinical and medical follow-up.

\section{Conflict of interest}

The authors declare no conflict of interest.

\section{References}

1. Laurent S, Cockcroft J, Van Bortel L, et al. Expert consensus document on arterial stiffness: methodological issues and clinical applications. Eur Heart J 2006; 27: 2588-605.

2. Boutouyrie P, Tropeano Al, Asmar R, et al. Aortic stiffness is an independent predictor of primary coronary events in hypertensive patients: a longitudinal study. Hypertension 2002; 39: 10-5.

3. Nichols WW, O'Rourke MF. McDonald's blood flow in arteries: theoretical, experimental and clinical principles. Edward Arnold, London 1990; 404-5.
4. Laurent S, Katsahian S, Fassot C, et al. Aortic stiffness is an independent predictor of fatal stroke in essential hypertension. Stroke 2003; 34: 1203-6.

5. Cruickshank K, Riste L, Anderson SG, et al. Aortic pulse wave velocity and its relationship to mortality in diabetes and glucose intolerance: an integrated index of vascular function. Circulation 2002; 106: 2085-90.

6. Antonini-Canterin F, Carerj S, Di Bello V, et al.; Research Group of the Italian Society of Cardiovascular Echography (SIEC). Arterial stiffness and ventricular stiffness: a couple of diseases or a coupling disease? A review from the cardiologist's point of view. Eur J Echocardiogr 2009; 10: 36-43.

7. Safar H, Mourad JJ, Safar M, et al. Aortic pulse wave velocity, an independent marker of cardiovascular risk. Arch Mal Coeur 2002; 95: 1215-8.

8. O'Rourke MF, Steassen JA, Vlachopoulus C, et al. Clinical applications of arterial stiffness: definitions and reference values. Am J Hypertens 2002; 15: 426-44.

9. Hermeling E, Reesink KD, Reneman RS, et al. Measurement of local pulse wave velocity: effects of signal processing on precision. Ultrasound Med Biol 2007; 33: 774-81.

10. Brands PJ, Hoeks AP, Willigers J, et al. An integrated system for the noninvasive assessment of vessel wall and hemodynamic properties of large arteries by means of ultrasound. Eur J Ultrasound 1999; 9: 257-66.

11. Hoeks AP, Brands PJ, Smeets FAM, et al. Assessment of the distensibility of superficial arteries. Ultrasound Med Biol 1990; 16: 121-8.

12. Kablak-Ziembicka A, Przewlocki T, Tracz W, et al. Diagnostic value of carotid intima-media thickness in indicating multi-level atherosclerosis. Atherosclerosis 2007; 193: 395-400.

13. Kasthalap V. Polyvascular atherosclerosis detection in patients with ST segment elevation myocardial infarction in prognosing the outcome of endovascular coronary revascularisation. Eurointervention 2010; 6 (Suppl. H): H54.

14. Mookadam F, Moustafa SE, Lester SJ, et al. Subclinical atherosclerosis: evolving role of carotid intima-media thickness. Prev Cardiol 2010; 13: 186-97.

15. Yeh ET, Anderson HV, Pasceri V, et al. C-reactive protein: linking inflammation to cardiovascular complications. Circulation 2001; 104: 974e546.

16. Haverkate F, Thompson SG, Pyke SD, et al. Production of C-reactive protein and risk of coronary events in stable and unstable angina. European Concerted Action on Thrombosis and Disabilities Angina Pectoris Study Group. Lancet 1997; 349: 462-6.

17. Danesh J, Lewington S, Thompson SG, et al. Plasma fibrinogen level and the risk of major cardiovascular diseases and nonvascular mortality: an individual participant meta-analysis. JAMA 2005; 294: 1799-809.

18. Kaptoge S, Di Angelantonio E, Pennells L, et al. C-reactive protein, fibrinogen, and cardiovascular disease prediction. N Engl J Med 2012; 367: 1310-20.

19. Paini A, Boutouyrie P, Calvet D, et al. Carotid and aortic stiffness: determinants of discrepancies. Hypertension 2006; 47: $371 \mathrm{e} 6$.

20. Libby P, Ridker PM, Maseri A. Inflammation and atherosclerosis. Circulation 2002; 105: 1135-43.

21. Blake GJ, Ridker PM. C-reactive protein and other inflammatory risk markers in acute coronary syndromes. J Am Coll Cardiol 2003; 41: 37-42.

22. Liang Y, Hou Y, Niu H, et al. Correlation of high-sensitivity C-reactive protein and carotid plaques with coronary artery disease in elderly patients. Exp Ther Med 2015; 10: 275-8. 
23. Stefanadi D, Tousoulis N, Papageorgiou A, et al. Inflammatory biomarkers predicting events in atherosclerosis. Curr Med Chem 2010; 17: 1690-707.

24. Karahan O, Acet H, Ertaş F, et al. The relationship between fibrinogen to albumin ratio and severity of coronary artery disease in patients with STEMI. Am J Emerg Med 2016; 34: 1037-42.

25. Kingwell BA, Waddell TK, Medley TL, et al. Large artery stiffness predicts ischemic threshold in patients with coronary artery disease. J Am Coll Cardiol 2002; 40: 773-9.

26. Hatsuda S, Shoji T, Shinohara K, et al. Regional arterial stiffness associated with ischemic heart disease in type 2 diabetes mellitus. J Atheroscler Thromb 2006; 13: 114-21.

27. Dart AM, Kingwell BA. Pulse pressure - a review of mechanisms and clinical relevance. J Am Coll Cardiol 2001; 37: 975-84.

28. Amar J, Ruidavets JB, Chamontin B, et al. Arterial stiffness and cardiovascular risk factors in a population-based study. J Hypertens 2001; 19: 381-7.

29. Sutton-Tyrrell K, Najjar SS, Boudreau RM, et al. Health ABC Study. Elevated aortic pulse wave velocity, a marker of arterial stiffness, predicts cardiovascular events in well-functioning older adults. Circulation 2005; 111: 3384-90.

30. Willum-Hansen T, Staessen JA, Torp-Pedersen C, et al. Prognostic value of aortic pulse wave velocity as index of arterial stiffness in the general population. Circulation 2006; 113: 664-70.

31. Vappou J, Luo J, Okajima K, et al. Aortic pulse wave velocity measured by pulse wave imaging (PWI): a comparison with applanation tonometry. Artery Res 2011; 5: 65-71.

32. Pannier BM, Avolio AP, Hoeks A, et al. Methods and devices for measuring arterial compliance in humans. Am J Hypertens 2002; 15: 743-53.

33. Wang Z, Yang Y, Yuan LJ, et al. Noninvasive method for measuring local pulse wave velocity by dual pulse wave Doppler. In vitro and in vivo studies. PLoS One 2015; 10: e0120482.

34. Gaszner B, Lenkey Z, Illyes M, et al. Comparison of aortic and carotid arterial stiffness parameters in patients with verified coronary artery. Disease Clin Cardiol 2012; 35: 26-31.

35. Horita Y, Tadokoro M, Taura K, et al. Relationship between carotid artery intima-media thickness and atherosclerotic renal artery stenosis in type 2 diabetes with hypertension. Kidney Blood Press Res 2002; 25: 255-9.

36. Morito N, Inoue Y, Urata M, et al. Increased carotid artery plaque score is an independent predictor of the presence and severity of coronary artery disease. J Cardiol 2008; 51: 25-32.

37. Pasierski T, Sosnowski C, Szulczyk A, et al. The role of ultrasonography of the peripheral arteries in diagnosing coronary artery disease. Pol Arch Med Wewn 2004; 111: 21-5.

38. Kablak-Ziembicka A, Przewlocki T, Ewa S, et al. Relationship between carotid intima-media thickness, cytokines, atherosclerosis extent and a two-year cardiovascular risk in patients with arteriosclerosis. Kardiol Pol 2011; 69: 1024-31.

39. Leone N, Ducimetiere P, Gariepy J, et al. Distension of the carotid artery and risk of coronary events: the three-city study. Arterioscler Thromb Vasc Biol 2008; 28: 1392e7.

40. Asmar R. Arterial stiffness and pulse wave velocity clinical applications. Elsevier, Paris 1999; 67, 101-2.

41. Blacher J, Safar ME, Pannier B, et al. Prognostic significance of arterial stiffness measurements in end-stage renal disease patients. Curr Opin Nephrol Hypertens 2002; 11: 629-34.

42. Sedaghat S, Mattace-Raso FU, Hoorn EJ, et al. Arterial stiffness and decline in kidney function. Clin J Am Soc Nephrol 2015; 10 2190-7.
43. Wilkinson IB, Franklin SS, Hall IR, et al. Pressure amplification explains why pulse pressure is unrelated to risk in young subjects. Hypertension 2001; 38: 1461-6. 